\title{
Low-Level Lead Exposure and Children's Intelligence From Recent Epidemiological Studies in the U.S.A. and Other Countries to Progress in Reducing Lead Exposure and Screening in the U.S.A.
}

\author{
Shigeo KOIKE
}

Department of Hygiene, Showa University School of Medicine, Tokyo

\begin{abstract}
From the 1980s many well-designed epidemiological studies have confirmed that low-level, subclinical lead exposure in early life is associated with decrements in children's intelligence. Neurodevelopmental deficits from exposure to a low level of lead have been held to be not only an American problem, but also a worldwide issue in the past decade. Good epidemiological studies were reported from England, Scotland, Germany, Greece, Australia and New Zealand.

Well-designed cross-sectional and prospective studies were carried out to quantify the magnitude of the relation between full scale IQ in children aged five years or more and the burden of lead ( $\mathrm{PbB}$ or $\mathrm{PbT}$ ) in early life of children. Of five crosssectional studies of blood lead, two demonstrated a significant inverse association between lead and IQ even after adjustment for confounders. Two other studies, however, showed no firm evidence of inverse association after adjustment for confounders, and the remaining study demonstrated no significant inverse association of five cross-sectional studies of tooth lead, two indicated an inverse association. between tooth lead and IQ, two others showed no significant inverse association after adjustment for confounders, and the remaining one manifested no association. Of four prospective studies, two revealed strong evidence of an inverse association between blood lead at the age of around two years and IQ. Another one, however, revealed an inverse association between mean postnatal blood level and IQ, while the remaining one demonstrated no significant inverse association between IQ and postnatal blood lead level after adjustment for confounders. In a comprehensive review of 26 epidemiological studies since 1979, including a meta-analysis, Pococok et al. indicated that doubling of the body lead burden (from 10 to $20 \mu \mathrm{g}$ /dl) blood lead or (from 5 to $10 \mu \mathrm{g} / \mathrm{g}$ ) tooth lead is typically associated with a mean deficit in full-seale IQ of around 1-2 IQ points.

Lead in interior household dust, exterior surface soil, and old residential lead paint, which is deteriorated or removed, constitute the major sources of lead poisoning in children in the United States. Infants and children, who typically engage in hand to mouth activities, frequently come into contact with lead dust in soil and on the floor. Marked declines both in air lead and blood lead concentrations are evident parallel to the phase-down of lead in gasoline and soldered cans by U. S. food processors. The major source of lead in drinking water is from lead pipes used in household plumbing. The CDC revised its guidelines concerning childhood lead poisoning, stating that community prevention activities should be triggered when a large percentage of children in a community have blood lead levels of $10 \mu \mathrm{g} / \mathrm{dl}$, the lowest level at which neurodevelopmental effects were believed to occur. For children with blood lead level concentrations between 10 and $14 \mu \mathrm{g} / \mathrm{dl}$, more frequent rescreening may be needed. For concentrations between 15 and $19 \mu \mathrm{g} / \mathrm{dl}$, in addition to more frequent screening, nutritional and educational advice should be given. In cases where these levels persist, there should be environmental investigation and intervention. All children with blood levels of $20 \mu \mathrm{g} / \mathrm{dl}$ or greater should receive environmental evaluation and medical examination. Such children may need pharmacological treatment.
\end{abstract}

Jpn J Hyg 1997 ; 52 : 552-61. 


\title{
Pulse Phenomenon of Continuous White Noise with Interrupted Pure Tone
}

\author{
Koichi MURATA ${ }^{* 1,2}$, Ryoichi INABA ${ }^{* 2}$ and Hirotoshi IWATA*2 \\ ${ }^{* 1}$ Course of Speech Pathology and Audiology, Nibon Chono Gengo Fukushi Gakuin, Nagoya \\ ${ }^{* 2}$ Department of Hygiene, Gifu University School of Medicine, Gifu
}

\begin{abstract}
The researches of masking made it clear that there is a bandpass like a filter in the auditory system. This is called the auditory filter and its central frequency is thought to be the frequency of an acoustic signal. If there exist this kind of filter, the authors expected that the noise image which is perceived when pure tone and noise simultaneously presented is different from the one which is perceived when noise is presented independently. The authors chose an interrupted pure tone as an acoustic signal and continuous white noise as noise, and experimented to investigate the pure tone effect on perception of white noise. As a result, two stimulus tones induced three sound images, which are sound images of continuous noise, a pure tone and pulsed noise. The sound image which listeners did not expect to hear was the pulsed noise image. This phenomenon was thought to be an auditory induction; the inducer is the sound image of the white noise presented with the silent part of pure tone of interrupted pure tone and the inducee is that of the white noise presented with pure tone part of interrupted pure tone. This pulse phenomenon suggests that pure tone changes the loudness of white noise. This finding is interesting for researches of auditory mechanism and for the field of hygienics with protecting the auditory system and improving hearing under the noisy environment.
\end{abstract}

Jpn J Hyg 1997 ; 52 : 562-6.

\section{Work Intensity During Working Hours and Different Types of Care Done by Special Nursing Home Workers}

\author{
Toshiko YOKOZEKI ${ }^{* 1}$, Jyunko WATANABE*2, Mituyo MAKITA*3 \\ Sachie HASUMURA*3, Miyoko HAMANO*2 and Jyoji FUJINAMI ${ }^{* 1}$ \\ ${ }^{* 1}$ Department of Priventive Medicine and Public Health, Tokyo Medical College, Tokyo \\ ${ }^{* 2}$ Department of Nutrition Education, Tokyo Kasei Gakuin University, Tokyo \\ ${ }^{* 3}$ Special nursing bome "Aizenen", Tokyo
}

\begin{abstract}
This study was done to estimated work intensity during working hours and different types of care to obtain basic data for making a care program. The subjects were care workers in good health ( $\mathrm{n}=8,24-45$ years) who worked in a special nursing home. The estimated maximal oxygen intake level, which is the maximal aerobic capacity, of each subject was assessed as normal to very good. The energy expenditure was $1787 \pm 534 \mathrm{kcal}$ during working hours. The work intensity was $0.061 \pm$ $0.011 \mathrm{kcal} / \mathrm{kg} / \mathrm{min}, 2.7 \pm 0.7 \mathrm{RMR}$, $98 \pm 6$ beats $/ \mathrm{min}$, and $30.3 \pm 2.0 \% \mathrm{Vo}_{2} \mathrm{max}$. Among the duties assessed for work intensity, bathing had the highest intensity, followed by transferring, changing diapers, feeding, and dressing. The work intensity of bathing was $0.081 \pm 0.031 \mathrm{kcal} / \mathrm{kg} / \mathrm{min}, 3.9 \pm 1.0 \mathrm{RMR}$, and $40.0 \pm 6.1 \% \mathrm{~V}_{\mathrm{O}_{2}} \mathrm{max}$, which was significantly higher than feeding, dressing, and transferring $(\mathrm{p}<0.05)$. Care giving at the time of bathing was significantly longer than the other care types $(\mathrm{p}<0.05)$. Feeding and transferring by inexperienced care workers were significantly low intensity $(\mathrm{p}<0.05)$. Work intensity of care was at high levels within the maximal permissible level in which fatigue doesn't make an appearance during working hours and in five types of care. Furthermore, care work intensity increased according to a decrease in the ADL level among the elderly. It is concluded that when making a care program, it is important to consider the ADL level of the elderly, work intensity and the amount of care-giving time, not only to maintain the health of care workers, but, also, to give superior quality care to the elderly.
\end{abstract}

Jpn J Hyg 1997 ; 52 : 567-73. 


\section{Environmental Health and Preventive Medicine Official Journal of the Japanese Society for Hygiene}

\section{INSTRUCTIONS FOR AUTHORS}

Environmental Health and Preventive Medicine as the official jour$\mathrm{nal}$ of the Japanese Society for Hygiene, is devoted to the publication of definitive studies including original papers, short communications and review articles, on human health sciences related to biological, physical, chemical, medical, psychosocial, and other various environmental factors.

\section{Categories of Publication}

Submissions may include :

(1) Original papers reporting results of original fundamental research in any branch in the environmental health and preventive medicine field,should conform to the Journal style.

(2) Short Communications reporting on research considered to require speedy publication, receive rapid review. They should be no more than 4 printed pages in length, containing an Abstract of about 100 words, a one-paragraph introduction, an abbreviated Materials and Methods section, Results and Discussion sections (which may be combined), a maximum of 20 references, and no more than 5 items for the display of date (any combination of tables and illustrations).

(3) Reviews on subjects relevant of the field. Submission of an outline of an unsolicited review may be approved by the Editorial Board, after which the complete article will be carefully evaluated before acceptance.

(4) Announcements of scientific meetings and of courses in Environmental Health and Preventive Medicine should be submitted at least 1 month prior to issue.

\section{Editorial Policies}

Submissions must be accompanied with the authors written confirmation that research contained in the parer has not been and will not be submitted to or published in any other scientific journal before a decision has been made as to its acceptability for publication in Environmental Health and Preventive Medicine. All authors listed on a manuscript must have agreed to its submission and content, as determined in the Uniform Requirements for Manuscripts Submitted to Biomedical Journals (JAMA1993;269;2282-6). Revisions to text or authors must be approved also by all authors. Environmental Health and Preventive Medicine accepts no responsibility for such changes. Associate Editors with broad expertise in all areas of Environmental Health and Preventive Medicine, along with a large group of external reviewers provide fair and thorough evaluation of papers submitted to Environmental Health and Preventive Medicine. Associate Editors and reviewers are expected to maintain a strict ethical code regarding confidentiality of the material under review. Any relation ships that could cause a conflict of interest with regard to a manuscript must by revealed by authors, reviewers, and Associate Editors to the Editor-in-Chief. After review, authors will be promptly informed of Editorial decision. Only written inquires regarding the status of manuscripts will be answered. Manuscripts only will be returned in the case of rejection. Accepted submissions become the property of the Japanese Society for Hygiene.

\section{Preparation of Manuscripts}

Original manuscripts must include :

(1) The author's covering letter containing the information in dupilate.

(2) Two copies of the manuscript and two sets of original illustrations. One set of original illustrations should be designated for use by the printer.

\section{Mailing Address}

Manuscripts should be sent to the Editorial Office, Human Research Inc., 2-5-16 Wakaba, Shinjuku-ku, Tokyo 160, Japan

\section{Cover Letter}

The paper should be submitted with a covering letter stating

(1) that the paper is being submitted for consideration for publication in Environmental Health and Preventive Medicine;

(2) the exact address for all related correspondence to be sent to and telephone and FAX numbers of the author;

(3) that there has been no previous publication of the paper or a similar paper and that no such publication is intended;

(4) agreement of all authors as to content and authorship of paper ;

(5) five key words describing the paper.

\section{Format and Style}

Manuscripts are to be written in clear, grammatical English. Papers that are not in Environmental Health and Preventive Medicine style or that are not in good idiomatic English will be returned to the author without review. Terminology and abbreviations not consistent with internationally accepted guidelines should be avoided.The manuscript should be typed on one side on ISO A4 paper with double spacing throughout, including in tables and illustration legends, with ample margins. Consecutive numbering of all pages is required, with the title page as page 1 . The manuscript should follow the order: (a) title, (b) authors and complete names and locations of institutions or laboratories, (c) running title, (d) key words, (e) footnotes, (f) abstract, ( $g$ ) text and references, (h) tables, (i) legends for all illustration, (j) illustrations and (k) other material.

\section{Title Page}

Title

Titles should be informative and brief, and limited to about 100 characters. Key words necessary to identify the nature of the subject matter must be included in the title.

Authors and Their Affiliations

Authors must include their full names, complete with first and middle names or initials. Author's academic degrees should not be included.The full names of institutions and subsidiary laboratories must by given for each affiliated author together with current addresses, including postal code. The name, complete address, telephone and FAX number of the author to whom proofs and correspondence are to be sent should be included separately on the title page.

Running Title vided.

A brief running title not to exceed 50 characters must be proKey Words

Five key words identifying the nature of the subject matter must be included on the title page. Footnotes to the title page text with superscript Arabic numerals are to be designated consecutively.

\section{Abstract}

The abstract should contain fewer than about 200 words yet be indicative of the content of the paper. It should indicate the purpose of the study, and the experimental technique, results, and interpretations of the date. All important terms relevant to the content of the paper should be incorporated into the abstract.Abbreviations should be kept to an absolute minimum; however, if they are needed,they must be properly identified so as to make the abstract independent of the text. 
ntroduction

Brief reference to papers relevant to the problem or question which the author's investigation adresses are sufficient.

\section{Materials and Metbods}

The methods should be briefly but clearly explained. Procedures published previously need only be cited in references. New and significant modifications need complete exposition. Papers re. porting human experimentation must be in line with principles of the Helsinki Declaration of 1975 , as revised in 1983. Papers involving human subjects must include a statement that informed consent was obtained from each subject or subject's guardian. Only results of those experiments in which proper attention has been given to experimental ethical considerations toward animals will be published. The statistical guidelines advocated by the International Committee of Medical Journal Editors (Ann Intern Med $1988 ; 108: 266-73)$ must be followed

Results

Results should concisely summarize the findings of the study and follow the train of thought presented in the methods section. Textual description of the date must be presented in tables and illustrations.

\section{Discussion}

Discussion should emphasize the major findings of the study and its significance. Information presented in the previous section must not be repeated. Concise interpretation of date with wellfounded speculation may by included.

\section{Abbreviations for Scientific Units}

Abbreviations for Scientific Units must conform to the System International (SI units).

\section{Acknowledgments}

Acknowledgments should specify information on technical help, material and financial support including the source(s) and number(s) of the grant (s).

\section{References}

References should be numbered in the order of their first mention in the text; cite only the number assigned to the reference. References should be double-spaced and be presented in numerical order. All references should be verified and checked that they have been cited in the text.Literature references must conform to the Uniform Requirements for Manuscripts Submitted to Biomedical Journals (JAMA $1993 ; 269: 2282-6$ ) as the following examples. For example :

(1) Papers published in Journals

Tarumi $\mathrm{K}$, Hagihara A, Morimoto $\mathrm{K}$. An investigation of effects lifestyle on care-seeking behavior using data from health insurance claims

Jpn J Hygiene 1995 ; 49 : 984-97.

(2) Books and other monographs

Grundy F. Preventive Medicine and Public Health. London : H. K. Lewis Co. Ltd., 1960.

(3) Contributions in Books

Waldman LK. Multidimensional measurements of regional strains in the intact heart. In : Glass L, Hunter P, McCulloch A, editors. Theory of Heart. New York; Springer-Verlag, 1991 : 145-74.

\section{Tables}

Tables of numerical data should be numbered and cited in numerical order in the text, and each be printed (also with doublespacing) on a separate page, numbered in sequence in Arabic numerals (Table 1,2 , etc.), provided with a heading, and referred to in the text as Table 1, Table 2, etc. Legends giving explanation sufficient to make the data intelligible without reference to the text must be typed on the same page.

\section{Illustrations and Illustration Legends}

Illustrations must be cited in the text in numerical order using Arabic numerals. Illustrations should be numbered in Arabic numerals according to the sequence of their appearance in the text, where they are referred to as Fig. 1, Fig. 2, etc. The name of the first author, the illustration number, and designation of the top of the illustration should be typed on a label adhered to the back of each illustration.Line drawings are to be submitted in either printouts from a laser printer,u or in camera-ready form on a white background, or in black ink. Illustration legends should be doublespaced on a separate sheet, and should include a brief title and a concise explanation of each illustration.

\section{Typesetting from Disks}

Environmental Health and Preventive Medicine is now copy editing accepted manuscripts electronically. Authors are encouraged to send a $31 / 2$ disk of the paper along with the required four hard copy printouts when submitting revised manuscripts. The entire manuscript should be contained in one file. Tables and mathematical material may be excluded from the disk file and presented only in the accompanying hard copy. Disks will not be returned to the authors. The disk should be labeled on the outside with Environmental Health and Preventive Medicine, the first author's name, a partial title of the manuscript, and the name of the computer file used to access the manuscript on disk. The name of the computer used (e.g., IBM/PS2), the name of the operating system and version (e.g. DOS 3.3), and the word processing program and version (e.g.Word Perfect 5.0) must be provided. The following word processing programs are preferred: Word Perfect (IBM or Macintosh), Word perfect for Windows, Microsoft Word (IBM or Macintosh), Mac Write II. Authors should not use the Fast Save option on Macintosh computers. Ensure that the letter " $\mathrm{i}$ "and the number " 1 " (also " $\mathrm{O}$ " and number " $O$ ") have been used properly, and format your article (tabs, indents, etc.) consistently. Characters not available on your word processor (Greek letters, mathematical symbols, etc.) should not be left open, but indicated by a unique code (e.g. @, \#, etc. for the Greek letters). Such codes must be used consistently throughout the text. The author should provide a list of such codes and a key. Do not allow your word processor to introduce word splits and do not use a 'justifed' layout. The Journal does not assume responsibility for errors in the conversion of newly released software, customized software, or special characters.

\section{Page Charge and Reprints}

No page charge is necessary for black-and-white printing. In the case of color photographs the cost of publishing will be borne by the authors. Reprints may be ordered at prices shown in the reprint order form which will be sent to the author.

\section{Proofs}

Proofs will be sent to authors without manuscripts. Only printing errors should be corrected. Changes in the manuscript will not be allowed. The corrected proofs must be returned within 48 hours after-receipt, preferably by FAX.

\section{Advertisements}

Advertisements must be received 5 weeks prior to the date of the issue in which the advertisement is to be published. Issues are published quarterly. Inquires regarding advertising should be directed to : Editorial Office, Department of Preventive Medicine and Public Health, Human Research Inc., 2.5-16 Wakaba, Shinjuku-ku, Tokyo 160, Japan. TEL: +81(3) 3358-4001 FAX: +81(3) 3358-4001. 